\title{
CONSTRAINT BASED NLP ENGINE
}

\author{
NABEEL SABIR KHAN ${ }^{1}$, ADNAN ABID ${ }^{1}$, MUHAMMAD SHOAIB \\ FAROOQ $^{1}$, YASER DAANIAL KHAN ${ }^{1}$, BILAL HASSAN ${ }^{1}$, AWAIS \\ KAMRAN $^{2}$, ANEESA ABBASI $^{2}$ \\ ${ }^{1}$ Department of Computer Science/SST, University of Management \& Technology \\ Lahore, Pakistan \\ \{nabeel.bloch, adnan.abid, shoaib.farooq, yaser.khan, bilal.hassan\}@ umt.edu.pk \\ ${ }^{2}$ Faculty of Information Technology, University of Central Punjab Lahore, Pakistan \\ \{kamranawais, aneesaabbasi\}@ucp.edu.pk
}

\begin{abstract}
Visual representations are always better than narrations in accordance to children, for better understanding. This is quite advantageous in learning school lessons and it eventually helps in engaging the children and enhancing their imaginative skills. Using natural language processing techniques and along the computer graphics it is possible to bridge the gap between these two individual fields, it will not only eliminate the existing manual labor involved instead it can also give rise to efficient and effective system frameworks that can form a foundation for complex applications. In this paper we present an architecture to design for a NLP engine that can be used for $3 D$ scene generation, the input would be in textual form that would be processed by each module of the natural language processing $(N L P)$ engine. This text would be restricted in terms of the constraint based grammar $(C B G)$, eliminating the maximum occurrence of any ambiguity and easing the noun fragmentation process. Eventually, the output of the NLP engine would be a sentence that fulfills the custom grammatical rules.

Keywords: NLP, Children, Stories, Visual Representation, Constraints, Image, Text to Scene
\end{abstract}

1. INTRODUCTION: The 3D tools that offer virtual scene generation mostly provide the users with the code based approach or the drag and drop approach. The complex interfaces and the their scripts make it difficult for the beginners to get used to them and a person with no domain knowledge of 3D engine and 3D coordinate system would find it even more difficult to use such a tool. Natural language driven software can offer a great deal of ease in usage, there are different advantages and disadvantages involved. The foremost advantage is that it may give rise to effective applications that can be driven through text or voice, and at the same side the disadvantage is the ambiguity involved in natural language. Though, there are different natural language techniques and algorithms available, some are based upon machine learning and some are heuristic based. Along with these techniques, eliminating the ambiguity and coming up with approaches to interpret semantics involved in the natural language text is the priority. The Ambiguity in natural language can only be handled if we consider a subset of the language instead of the whole language itself, this will filter such texts with no proper meaning. In terms of mapping text onto the scene, this would be a lot beneficialas only such sentences would be mapped that actually hold a spatial representational meaning and the rest would be discarded. This brings out the idea to of restricting grammar on some solid basis, which is followed in the section (3). We propose the following system architecture for the NLP engine. 


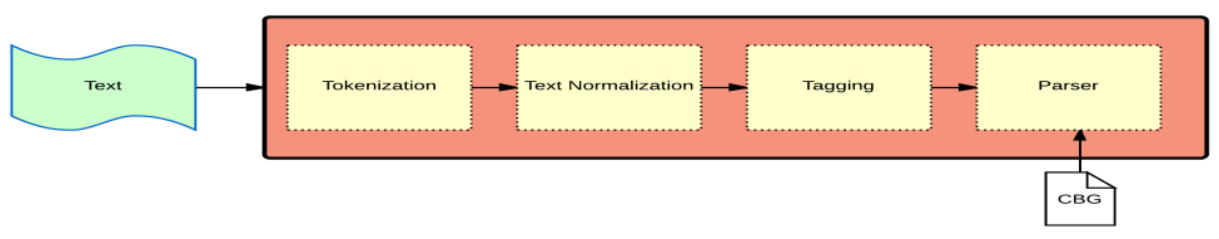

Figure 1: Constraint based NLP Engine Architecture

Text serves as an input, which is processed through various natural language modules. The processed text is later used for substantiating its syntax with the help of the parser driven by the Constraint based grammar, this would validate the sentence in terms of its meaning in terms of spatial domain that can be either a 3-dimensional or 2dimensional.Moreover, this NLP engine can be extended to game prototypingas well, in terms of building up a 3D scenes through simplified textual descriptions. With such a system, game prototypes can be generated with ease with objects and their placement defined just like the way we imagine it along with custom delineation. The 2 dimensional comic application and cartoon animation software can also make a good use of such a framework, in providing a medium of natural language to initiate their processing. Though, the scope of our work is confined to simple storied sentence structures, for this purpose, we have collected stories written by primary level students in order to determine the complexity of the sentence structure that we'll be using as the input and to deduce highlighted grammatical rules.

2. RELATED WORK: The efforts were started in early 90's. The project was named as SHRDLU which was a system capable of participating in a dialogue about a micro world (the blocks world) and manipulating this world according to commands issued in English by the user [5]. LUNAR and SHRDLU both exploited the limitations of the domain to make the natural language understanding problem tractable. It was basically a language parser and handled a narrow set of grammar rules and vocabulary sentences. It was a successful project in terms of comprehending the semantics out of the natural language text. Another system of image generation from natural language was by G. Adorni et all [6]with a limitation that it only accepts a simple format of sentences based upon few parts of speech components. It was very simple implementation but recognized as one of the foundation steps in the field. The Put System [16] was a system that was meant to make the scene generation easier but it was fairly restricted to the spatial arrangements of objects. The constraint that it introduced was the input in the form of PUT $(\mathrm{X}, \mathrm{P}, \mathrm{Y})^{+}{ }^{+}$where $\mathrm{X}$ and $\mathrm{Y}$ were the objects and $\mathrm{P}$ represented the spatial preposition.

CARSIM project introduced by Benekohal $\mathrm{R} F$ et all was another effort for getting visual representation of the road accidents [7]. However, one of the most appreciated work was WordsEye as discussed above [4]. The task of 3D scene generation from the combination of voice and text is performed by Seversky et all [8]. Their work is very good example of human computer interaction but in our case we are only concerned with the text as input.

The work of inspiration for us was NLP story Maker by Takako Aikawa et all [9]. It was too good but again following the grammar rules strictly which is not a suitable choice for a text to scene generation API designed for children because one would always come across certain sentences which does not hold any spatial meaning in terms 3D domain, hence restricting the grammar is inevitable.

Almost all of the above discussed systems follow grammatical rules somehow, we have restricted the grammar in our framework because the sentences written by the school goings are not strict towards the grammar rules or vocabulary, as at an early stage a primary level student is not well accustomed with all grammatical rules and spellings. However, their sentence structures are mainly based mostly upon the use of prepositions [10] and certain other grammar components, which were analyzed and deductions were made in terms of the coming up with a grammar, for the parser we used keeping the spatial understanding intact. That's why in our work we have defined our own grammar rules on the basis of preposition, verbs for semantics and nouns majorly along with adjectives that 
help in depicting the objects. The details of stories collected by us and defined grammar are coming in the next sections.

3. COLLECTION AND ANALYSIS OF STORIES: We collected some stories from children of a particular age group variation from six to eight years and some of the famous fairy tales written for such students to develop our dataset. The students of this age group are much familiar about basic story writing skills. The stories comprises of 3 to 7 English language sentences as shown in the figure 1.0, 1.1 and 1.2. After going through the aforementioned stories, selective sentences they were tagged using the Penn Tree bank [17] using Apache OpenNLP machine learning based natural language processing toolkit [18]. After tagged tokens were obtained from the sentences of our data set, they were analyzed in terms of frequency distribution of each tag as shown in figure 1.3, this helped in identifying tags that would be a need in coming up with a custom grammar. The results showed the excessive use of the nouns $(21.75 \%)$, verb $(25.54 \%)$, prepositions $(2.48 \%)$, adverbs $(8.02 \%)$, adjectives $(10.07 \%)$ and personal pronouns $(13.87 \%)$.

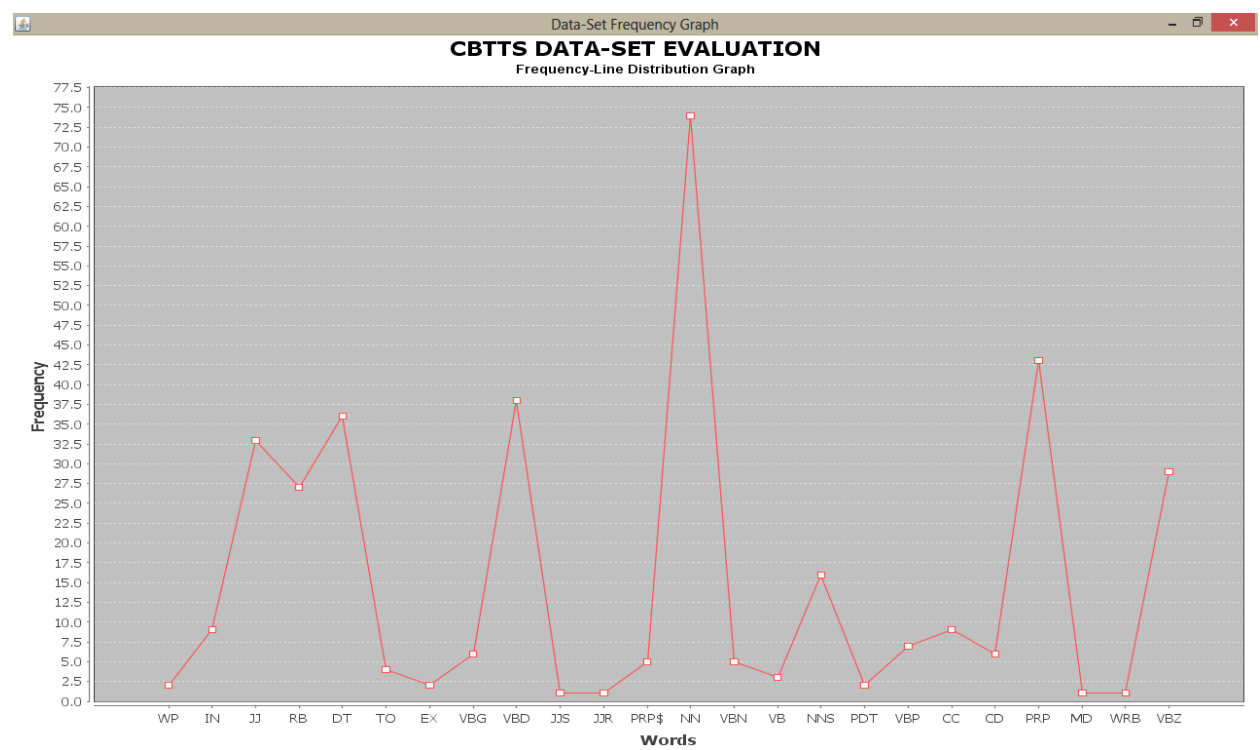

Figure 2: Frequency Distribution Grpah for Tagged Tokens of the Dataset

4. DEVELOPMENT OF CBG (Constraint Based Grammar):At this stage of analysis, it will simply be determined that what tags would dominate the custom grammar along which of the tags would actually make sense for example all the tags corresponding to \{What, Where, How, Who, Whose, \} that gave rise to a question statements were eliminated because they don't hold a representational meaning. The idea was not to eliminate the low frequency tags and not use them in defining our own custom grammar, instead it was important to analyze the combination of tags that were mostly used in a sentence structure.

Dealing with spatial representation, nouns are the most important of all, without them there won't be any object to refer or place in the 3D domain, on the other hand for semantics verbs were important. Conditional probabilities were calculated for each valid combinations, and are presented in figure 1.2 and 1.3.

Given that all the verb tags, VB (verb base form), VBD (verb past tense), VBZ (verb $3^{\text {rd }}$ person present), VBN (Verb past participle), VBG (Verb present participle) and VBP (Verb non- $3^{\text {rd }}$ person singular present) fall under the production of Verb, as used in the figures below.

$$
\mathrm{Verb} \rightarrow \mathrm{VB}|\mathrm{VBD}| \mathrm{VBZ}|\mathrm{VBG}| \mathrm{VBP} \mid \mathrm{VBN}
$$




$$
\rho\left(T A G_{X} \mid T A G_{Y}\right)=\frac{\rho\left(T A G_{Y} \cap T A G_{X}\right)}{\rho\left(T A G_{Y}\right)}
$$

\section{Equation 1: Conditional probability of Tags}

This form of analysis is inspired by the concept of Bi-gram model [24], where further two combination of tags would be analyzed to deduce details for Nouns and Verb productions to structure our own grammar. Noam Chomsky composed a sentence in 1957 [25], "colorless green ideas sleep furiously" this refers to the correct grammatical structure but false semantics. Many such sentences can be formed which are wrong semantically, our assumption in accordance for this system is that restricting input in terms of grammar, Objects and semantics may help in solving the issues of language ambiguity.

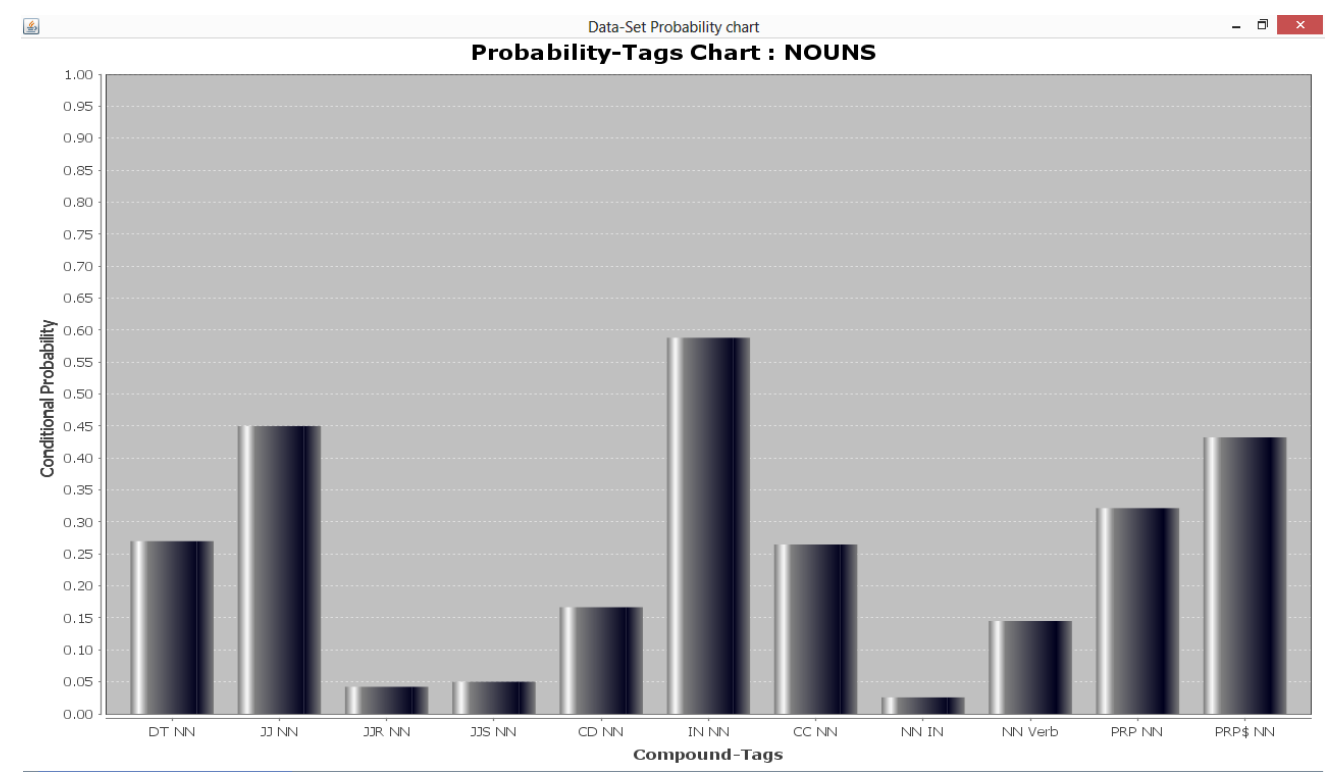

Figure 3: Conditional Probabilities of Tagged Noun Combinations 
Conclusion \& Future Work: In our work, we have developed a text to scene generation system for the school going kid's story writing. The objective of this system is to aid the understanding capabilities of the kids of a particular age group. To the best of our knowledge, the system is a novel contribution for the targeted audience of this particular age group. We collected the written stories from the kids. We have combined the techniques from natural language processing and computer graphics and presented a hybrid model. We have defined a custom set of grammar rules and prepositions. We have tested our developed system on the stories written by students. Apart from that, we are missing the full use of English language grammar and preposition. We have limited set of objects stored in our dataset although more objects can be added manually. However, the need for automated addition of objects is desired one. We are hopeful that this system will be like a fun game for the kids which will defiantly increase their interest in learning and understanding.

\section{REFERENCES}

[1] Mayer, R. E. (1997). Multimedia learning: Are we asking the right questions?.Educational psychologist, 32(1), $1-19$.

[2] Pashler, H., McDaniel, M., Rohrer, D., \& Bjork, R. (2008). Learning styles concepts and evidence. Psychological science in the public interest, 9(3), 105-119.

[3] Stokes, S. (2002). Visual literacy in teaching and learning: A literature perspective. Electronic Journal for the Integration of technology in Education, 1(1), 10-19.

[4] Coyne, B., \& Sproat, R. (2001, August). WordsEye: an automatic text-to-scene conversion system. In Proceedings of the 28th annual conference on Computer graphics and interactive techniques (pp. 487-496). ACM.

[5] Winograd, T. (1971). Procedures as a representation for data in a computer program for understanding natural language.

[6] Adorni, G., Di Manzo, M., \& Ferrari, G. (1983, September). Natural language input for scene generation. In Proceedings of the first conference on European chapter of the Association for Computational Linguistics (pp. 175-182). Association for Computational Linguistics.

[7] Taylor, A., Marcus, M., \& Santorini, B. (2003). The Penn treebank: an overview. In Treebanks (pp. 5-22). Springer Netherlands.

[8] Seversky, L. M., \& Yin, L. (2006, October). Real-time automatic 3D scene generation from natural language voice and text descriptions. In Proceedings of the 14th annual ACM international conference on Multimedia (pp. 61-64). ACM.

[9] Aikawa, T., Schwartz, L., \& Pahud, M. NLP Story Maker.

[10] List of English Language Prepositions Accessed Dec, 2013

[11] [Available] http://www.englishclub.com/grammar/prepositions-list.htm

[12] S. R. Clay and J. Wilhelms,(1996, March) "Put: Language-Based Interactive Manipulation of Objects", IEEE Computer Graphics and Applications, pages 31-39,.

[13] Penn Tree Bank Tag set, [Available] https://www.ling.upenn.edu/courses/Fall_2003/ling001/penn_treebank_pos.html

[14] Apache OpenNLP machine learning based toolkit for natural language processing [Available] https://opennlp.apache.org/

[15] Bi-gram Language Model [Available] http://www.ee.columbia.edu/ln/LabROSA/doc/HTKBook21/node132.html

[16] Chomsky's point [Available] https://www.princeton.edu/ achaney/tmve/wiki100k/docs/Colorless_green_ideas_sleep_furiously.html 